\title{
Multi-User Subcarrier Number Modulation-based OFDM for Future Wireless Communication Networks
}

\author{
MUHAMMET KIRIK ${ }^{1}$, JEHAD M. HAMAMREH ${ }^{1}$ \\ ${ }^{1}$ M. Kirik and J. M. Hamamreh are with WISLAB for Wireless Research at the Department of Electrical-Electronics Engineering, Antalya Bilim University, \\ Antalya, 07190, Turkey. (web: https://sites.google.com/view/wislab). \\ Corresponding author: Muhammet Kirik, email: muhammetkirik1997@gmail.com.
}

The Matlab simulation codes used to generate the results in this paper can be found at https://researcherstore.com

\begin{abstract}
Orthogonal Frequency Division Multiplexing with Subcarrier Number Modulation (OFDMSNM) has recently been proposed as an effective transmission method that can transmit additional data bits by exploiting the number of subcarriers in each subblock. This results in an improved performance in terms of spectral efficiency and reliability. However, one of the main drawbacks of OFDM-SNM is that not all the available subcarriers are deployed to transmit data, as some of these subcarriers remain inactive. In order to eliminate this problem and make use of all the available subcarriers, Multi-User OFDM-SNM is proposed in this paper for serving multiple users by dedicating the subcarriers used for implementing SNM to serve a far user, whereas the remaining subcarriers are used to send data for a near user. In this paper, the concept of multi-user OFDM-SNM is established on the basis of conventional OFDM over a Rayleigh fading channel. The validity of the system is proven by exhibiting both theoretical analysis and computer simulations. The obtained results show that the proposed multi-user OFDM-SNM is a strong candidate for the future $6 \mathrm{G}$ and beyond technologies and it can satisfy the requirements of multi-user cases of future wireless systems demanding higher reliability and better spectral efficiency.
\end{abstract}

INDEX TERMS OFDM, OFDM-SNM, subcarrier number modulation, wireless communication, 5G, 6G, multi-user, future wireless networks.

\section{INTRODUCTION}

W ITH the advancement in the Internet of Things (IoT) technologies and the increasing demand from Internet applications and services, merits as high data rate, high spectral efficiency (SE), and high data reliability have started to receive more attention than ever to satisfy the diverse necessities of future $5 \mathrm{G}, 6 \mathrm{G}$, and beyond technologies [1][4].

One of the biggest impediments against the high data rate capability of future wireless communication systems is the intersymbol interference (ISI) problem [5]-[7]. Due to their ability in reasonably minimizing the effect of this problem, multicarrier systems such as OFDM have received great interest in recent years [8], [9]. By adding a guard interval known as the cyclic prefix (CP) to each OFDM block and arranging the subcarrier spacing according to the channel coherence bandwidth of the system, it has been seen that each subcarrier can be located orthogonal to other subcarriers and the ISI can be abolished [10], [11]. With the merits of OFDM, such as easy equalization, multi-user adaptivity, and availability for different modulation techniques [12], it is considered to be one of the most promising techniques for many of the communication standards [13]. However, conventional OFDM suffers from some certain limitations such as high peak-to-average power ratio (PAPR) [14], [15], and its plain performance is not enough to satisfy the requirements of current demands in terms of reliability and SE. Due to this situation, OFDM requires improved modulation techniques to increase the performance of the aforementioned metrics [16].

To overcome the spectral efficiency issue, researchers have come up with different modulation techniques paired with OFDM, such as OFDM with index modulation (OFDMIM) [17], OFDM with subcarrier power modulation (OFDM- 
SPM) [18], [19], and OFDM with subcarrier number modulation (OFDM-SNM) [20].

OFDM-IM is a technique that can transmit data bits by exploiting not only conventional $M$-ary signal constellations but also exploiting the indices of the active subcarriers in each subblock to increase the spectral efficiency and reliability [21]. OFDM-SPM is another technique which focuses on the transmission of additional data bits along with the conventional $M$-ary modulated bits by utilizing the power of subcarriers in OFDM blocks as a third dimension [19], [22]-[27]. On the other hand, OFDM-SNM is a technique that focuses on the improvement of the spectral efficiency and reliability performance by exploiting the number of subcarriers in each subblock to transmit additional data bits along with those bits that are modulated by the conventional $M$-ary modulation scheme [20]. Even though these modulation techniques and many others for both OFDM systems have aroused great interest and numerous works have been done based on them such as [4], [28]-[36], there is a gap in these techniques' multi-user capabilities, which remains as an intriguing research problem [37].

In this regard, a novel multi-user adaptive OFDM-SNM scheme is proposed in this paper. In this scheme, it is aimed to serve two users simultaneously, who are located in a far position and a near position to the transmitter. While the user, who is located in a far location from the transmitter is served by exploiting the merits of OFDM-SNM, which relies on the idea of activating the subcarriers according to incoming data of the far user for data transmission, another user, who is located in a near location is aimed to be served by those subcarriers that are not deployed for the transmission of the far user. The main contributions of the proposed scheme can be summarized as follows:

- The proposed transmission technique results in obtaining high spectral efficiency and low BER by avoiding the waste of subcarriers that remain inactive during the operation of OFDM-SNM and creates an opportunity to make use of those inactive subcarriers by another user.

- Since OFDM-IM dictates the indices of the active subcarriers to transmit additional data bits, it suffers from the inability to make the selection of the subcarriers to be channel-dependent as the selected subcarriers' indices are purely data-dependent. However, in OFDMSNM, the selection of the active subcarriers can be operated by the number of subcarriers, which indicates the data dependency, and also these active subcarriers can be chosen from those subcarriers that have the highest channel capacity. Thus the proposed scheme can operate the transmission in a state being both channel and data dependent at the same time.

- The proposed multi-user scheme can also be adapted to the other OFDM based modulation techniques such as OFDM-IM, OFDM-SPM, and OFDM with subcarrier gap modulation (OFDM-SGM) [38], since all these schemes are suffering from the same drawback, which is not deploying all the available subcarriers to transmit data, as some of these subcarriers remain inactive and lead to a waste of spectrum.

Consequently, in this paper, a strong scheme is proposed as a potential candidate for the future $5 \mathrm{G}, 6 \mathrm{G}$, and beyond wireless networks that require multi-user services.

The remaining parts of this paper are organized as follows. In section II, the system model of the proposed multi-user OFDM-SNM scheme is comprehensively explained in detail. In section III, the performance analysis of the proposed system is provided. In section IV, the simulation results are exhibited and explained. Lastly, with section V, the paper is concluded. ${ }^{1}$

\section{SYSTEM MODEL}

The transmission structure of the proposed multi-user OFDM-SNM scheme is given in Fig. 1. The transmission starts with with the data of the far user, which is defined as $m$ bits, entering the system. These $m$ bits are split into $G$ groups in which each of them contain $p=p_{1}+p_{2}$ bits. These $G$ groups are deployed to determine the length of the OFDM subblocks, $N=N_{F} / G$, where the $N_{F}$ is the size of the Fast Fourier Transform (FFT). In each $p=p_{1}+p_{2}$ bits, $p_{1}$ represents the SNM mapper, which defines the number of subcarriers that will be activated for the transmission of the data modulated by the conventional $M$-ary modulation, named as $p_{2}$.

It should be noted that on the contrary to OFDM-IM, the proposed scheme does not dictate any certain number of active subcarriers, $K$, for the transmission. However, the possible values of $K$ vary according to the $p_{1}=\log _{2}(N)$ out of $N$ number of available subcarriers in the system, which leads the value of $K$ to be $K \in[1,2, \ldots, N]$. Since $K$ does not have a fixed value, and it is dependent on the incoming SNM mapper bits, $p_{1}$, the subcarrier activation and the defined value of $K$ may differ for different cases. For such a case where $N=4, K \in[1,2,3,4]$, and $p_{1}=\log _{2}(4)=2$, Table I shows the possible values of $K$ according to $p_{1}$.

TABLE 1. SNM mapper with $p_{1}=2 \& N=4$

\begin{tabular}{|l|l|l|}
\hline $\begin{array}{l}\text { Information } \\
\text { bits }\left(p_{1}\right)\end{array}$ & $\begin{array}{l}\text { Active Subcarriers } \\
\text { for the Far User }\end{array}$ & $\begin{array}{l}\text { Active Subcarriers } \\
\text { for the Near User }\end{array}$ \\
\hline$\left[\begin{array}{ll}0 & 0\end{array}\right]$ & {$\left[\begin{array}{llll}1 & 0 & 0 & 0\end{array}\right]$} & {$\left[\begin{array}{llll}0 & 1 & 1 & 1\end{array}\right]$} \\
\hline$\left[\begin{array}{ll}0 & 1\end{array}\right]$ & {$\left[\begin{array}{llll}1 & 1 & 0 & 0\end{array}\right]$} & {$\left[\begin{array}{llll}0 & 0 & 1 & 1\end{array}\right]$} \\
\hline$\left[\begin{array}{ll}1 & 0\end{array}\right]$ & {$\left[\begin{array}{llll}1 & 1 & 1 & 0\end{array}\right]$} & {$\left[\begin{array}{llll}0 & 0 & 0 & 1\end{array}\right]$} \\
\hline$\left[\begin{array}{ll}1 & 1\end{array}\right]$ & {$\left[\begin{array}{llll}1 & 1 & 1 & 1\end{array}\right]$} & {$\left[\begin{array}{llll}0 & 0 & 0 & 0\end{array}\right]$} \\
\hline
\end{tabular}

According to this lookup table, for each subblock the remaining $p_{2}=K\left(\log _{2}(M)\right)$ bits are modulated by the $M$-ary signal constellation to transmit the data symbols of the far user over the active subcarriers. After this process is

\footnotetext{
${ }^{1}$ Notation: Bold, lowercase, and capital letters are used for column vectors and matrices, respectively. (.)H represents Hermitian transposition. $\operatorname{det}(\mathrm{A})$, $\operatorname{det}(\mathrm{B}) \operatorname{denote}$ the determinant of $\mathrm{A}$ and $\mathrm{B}$ respectively. $\circledast$ denotes a circular convolution operation.
} 


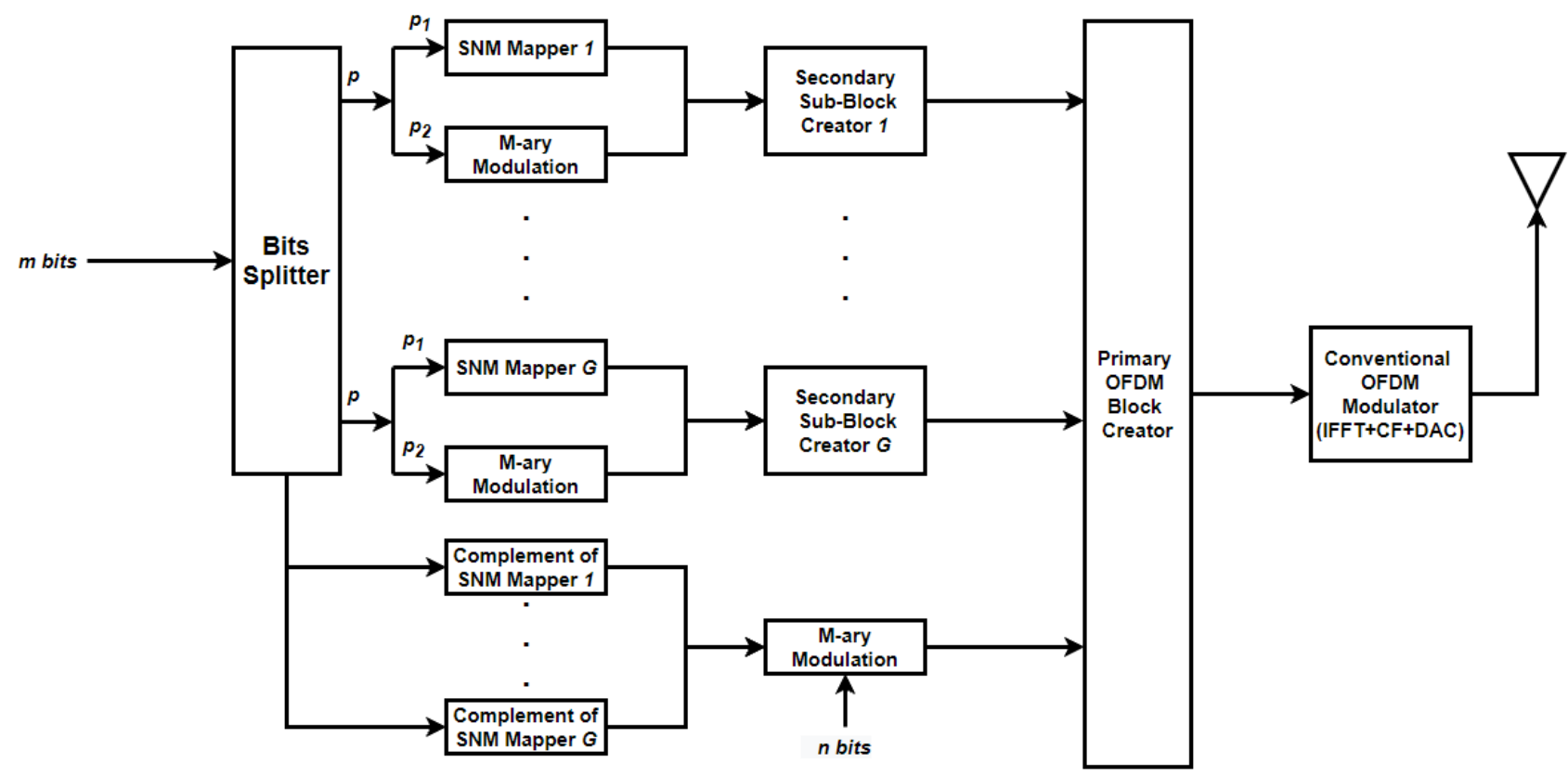

FIGURE 1. Transmitter Structure of the Proposed Multi-User OFDM-SNM.

applied on all the $G$ subblocks the primary OFDM block can be represented as

$$
\mathbf{x}_{\mathbf{F}_{\mathbf{f}}}=\left[x_{F_{f}}(1), x_{F_{f}}(2), \ldots, x_{F_{f}}\left(N_{F}\right)\right] .
$$

While this process guarantees the transmission of the data of the far user on the one hand, on the other hand, the transmission of the near user's data is also initiated. For each symbol of $p_{2}$ bits transmitted to the far user, the remaining subcarriers are deployed to transmit each symbol of the near user's data, $n$. In order to operate this transmission, $(N-K)$ number of subcarriers are deployed to keep the unemployed subcarriers of OFDM-SNM active to prevent the waste of spectrum. Table I shows the activation pattern of the subcarriers that are deployed for the transmission of the near user's data. As each of the symbols of $n$ bits is assigned to be conveyed over $(N-K)$ subcarriers by $p_{1}, n$ is modulated by conventional $M$-ary modulation. After this process is applied on all the $G$ subblocks the primary OFDM block can be represented as

$$
\mathbf{x}_{\mathbf{F}_{\mathbf{n}}}=\left[x_{F_{n}}(1), x_{F_{n}}(2), \ldots, x_{F_{n}}\left(N_{F}\right)\right] .
$$

As an example case, Fig. 2 shows a simple operation of the transmission for both the far and near users. Since $p_{1}$ is 10 , the transmission of $p_{2}$, which is 0 , must be operated by three subcarriers. After the transmission of $p_{2}$ is achieved by these three subcarriers, the remaining subcarrier is deployed to convey the symbol of the secondary incoming data stream, 1 , that is sent to the near user.

After this process has been completed for the far and near users, the remaining steps are operated the same as the conventional OFDM modulation for both users. By taking the inverse fast Fourier transform (IFFT) to $\mathbf{x}_{\mathbf{F}_{\mathbf{f}}}$ and $\mathbf{x}_{\mathbf{F}_{\mathbf{n}}}$ separately, the output vectors $\mathbf{x}_{\mathbf{t}_{\mathbf{f}}}$ and $\mathbf{x}_{\mathbf{t}_{\mathbf{n}}}$ are acquired with dimension $N_{F} \times 1$. CP of length $\left(N_{C P}\right)$ is appended on both groups of data to alleviate the inter symbol interference (ISI) effect. As a result, the output signals for the far and near users respectively become

$$
\begin{aligned}
& \mathbf{x}_{\mathbf{C} \mathbf{P}_{\mathbf{f}}}=\left[\mathbf{x}_{\mathbf{t}_{\mathbf{f}}}\left(N_{F}-N_{C P}+1: N_{F}\right) \mathbf{x}_{\mathbf{F}_{\mathbf{f}}}\right], \\
& \mathbf{x}_{\mathbf{C P}_{\mathbf{n}}}=\left[\mathbf{x}_{\mathbf{t}_{\mathbf{n}}}\left(N_{F}-N_{C P}+1: N_{F}\right) \mathbf{x}_{\mathbf{F}_{\mathbf{n}}}\right] .
\end{aligned}
$$

After this point, the output signals in time domain are transmitted over the multipath Rayleigh fading channels by the channel impulse response (CIR), $\mathbf{h}_{\mathbf{t}_{\mathbf{f}}}=\left[h_{t_{f}}(1), h_{t_{f}}(2), \ldots, h_{t_{f}}(v)\right]$ and, $\mathbf{h}_{\mathbf{t}_{\mathbf{n}}}=$ $\left[h_{t_{n}}(1), h_{t_{n}}(2), \ldots, h_{t_{n}}(v)\right]$ where $v$ is the length of CIR, and these output signals are affected by the additive white Gaussian noise (AWGN) with the noise variance of $N_{0, T}$ in the time domain to obtain the received signals of the far and near users. These procedures are mathematically represented for both the far and near users respectively as

$$
\begin{gathered}
\mathbf{y}_{\mathbf{t}_{\mathrm{f}}}=\mathbf{x}_{\mathbf{t}_{\mathrm{f}}} \circledast \mathbf{h}_{\mathbf{t}_{\mathrm{f}}}+\mathbf{n}_{\mathbf{z}_{\mathrm{f}}}, \\
\mathbf{y}_{\mathbf{t}_{\mathrm{n}}}=\mathbf{x}_{\mathbf{t}_{\mathrm{n}}} \circledast \mathbf{h}_{\mathbf{t}_{\mathrm{n}}}+\mathbf{n}_{\mathbf{z}_{\mathrm{n}}},
\end{gathered}
$$

where $\mathbf{n}_{\mathbf{z}_{\mathbf{f}}}$ and $\mathbf{n}_{\mathbf{z}_{\mathbf{n}}}$ are the AWGN vectors.

The receiver of the proposed multi-user OFDM-SNM reverses the operations done at the transmitter by removing $\mathrm{CP}$, performing FFT, SNM demapping, for only the far user's receiver, and detection. After the $\mathrm{CP}$ is removed from the 


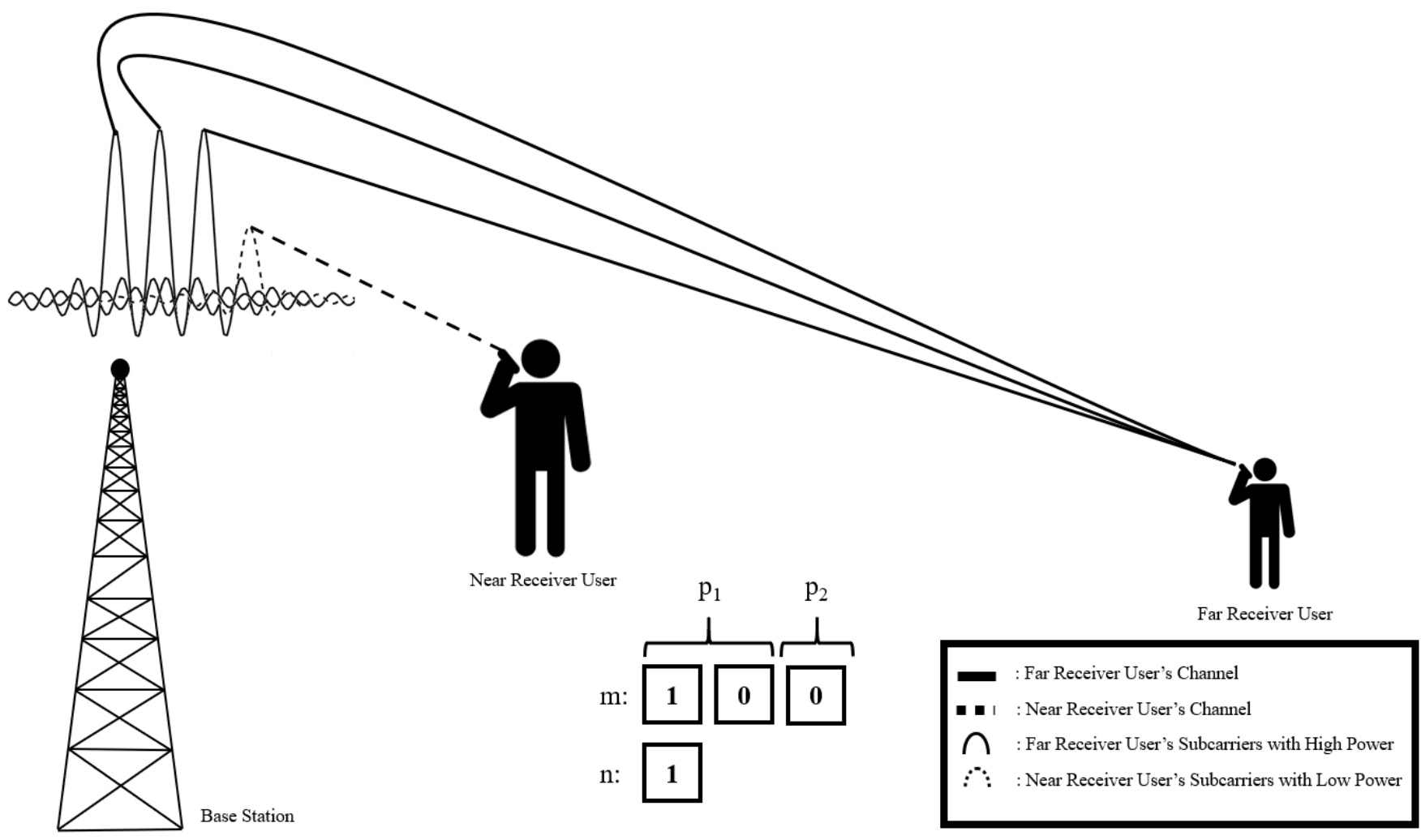

FIGURE 2. A sample case to illustrate the transmission of the far user's and near user's data, where $p_{1}$ is 10 .

received signals, the acquired signals for the far and near users become

$$
\begin{aligned}
\mathbf{y}_{\mathbf{t}_{\mathbf{f}}} & =\left[\begin{array}{llll}
y_{0_{f}} & y_{1_{f}} & \ldots & y_{\left(N_{F}-1\right)_{f}}
\end{array}\right], \\
\mathbf{y}_{\mathbf{t}_{\mathbf{n}}} & =\left[\begin{array}{llll}
y_{0_{n}} & y_{1_{n}} & \cdots & y_{\left(N_{F}-1\right)_{n}}
\end{array}\right],
\end{aligned}
$$

respectively, while after performing the FFT they become

$$
\begin{gathered}
\mathbf{y}_{\mathbf{f}}=\left[\begin{array}{llll}
y_{F_{f}}(0) & y_{F_{f}}(1) & \ldots & y_{F_{f}}\left(N_{F}-1\right)
\end{array}\right], \\
\mathbf{y}_{\mathbf{n}}=\left[\begin{array}{llll}
y_{F_{n}}(0) & y_{F_{n}}(1) & \ldots & y_{F_{n}}\left(N_{F}-1\right)
\end{array}\right] .
\end{gathered}
$$

After this point, a simple frequency domain equalizer is applied to both sets of output data. In the next step, a simple spatial energy based detector is deployed to extract the pattern of the active subcarriers by selecting an appropriate threshold value for the far user. The reason why this type of a detector is used in the proposed system is to facilitate simplicity, rather than using complex detectors such as ML or LLR detectors [39], [40]. After that, the number of active subcarriers is defined in each subblock and their corresponding bits are determined by the SNM demapper, which is the reverse process of SNM mapper at the transmitter to obtain additional data bits of the far user. Now, the active subcarriers obtained at the receiver for each subblock are used for the detection of the constellation symbols. As the last step of the reception of the far user, all the bits that come from the SNM demapper and the symbol detection are merged for each subblock. By doing this procedure for all the subblocks the reception of the far user's data is concluded. Since there is no need to demap SNM for the reception of the near user, the constellation detection of the near user's data is operated conventionally for each subblock and by doing the same procedure for all the subblocks the received data of the near user is acquired for the whole OFDM block.

\section{PERFORMANCE ANALYSIS}

In this section, the proposed multi-user OFDM-SNM scheme's performance is analyzed in terms of spectral efficiency, bit error probability, and power efficiency as follows.

\section{A. SPECTRAL EFFICIENCY (SE)}

The formulated SE (bits/s/Hz) derivations of the proposed multi-user OFDM-SNM for the far and near users are given respectively as follows:

$$
\begin{gathered}
\eta_{\text {Far }}=\frac{\sum_{g=1}^{G}\left(\log _{2}(N)+K(g) \log _{2}(M)\right)}{N_{F}+N_{C P}}, \\
\eta_{N \text { ear }}=\frac{\sum_{g=1}^{G}\left(\log _{2}(N)+((N-K)(g)) \log _{2}(M)\right)}{N_{F}+N_{C P}} .
\end{gathered}
$$


In these formulas, $K(g)$ represents the number of active subcarriers that are selected out of $N$ subcarriers for the transmission of the far user's data. As it can be inferred from the derived formula of the near user, however, for the near user's data, the SE is calculated by exploiting the $(N-K)(g)$, which represents the number of subcarriers that are not activated for the transmission of the far user's data but activated for the transmission of the near user's data instead.

\section{B. ANALYTICAL BIT ERROR PROBABILITY}

At the two different reception sides of the proposed scheme, there are four possibilities of detection for the transmitted data of the far and near users, which are the correct detection $X_{f}, X_{n}$ and erroneous detection $\hat{X}_{f}, \hat{X}_{n}$. Thus, for the detection of the transmitted data at both the far user's and near user's reception end, the BER considers two different pairwise error probabilities, $P\left(X_{f}-\hat{X}_{f}\right)$ and $P\left(X_{n}-\hat{X}_{n}\right)$. In this model, the frequency selective channel is considered to be fixed within one block and it follows a Rayleigh distribution. The transmitted data of the far and near users are formulated respectively in the frequency domain as follows:

$$
\begin{gathered}
\mathbf{y}_{\mathbf{f}}=\mathbf{X}_{\mathbf{f}} \mathbf{h}_{\mathbf{f}}+\mathbf{n}_{\mathbf{z}_{\mathbf{f}}}, \\
\mathbf{y}_{\mathbf{n}}=\mathbf{X}_{\mathbf{n}} \mathbf{h}_{\mathbf{n}}+\mathbf{n}_{\mathbf{z}_{\mathbf{n}}} .
\end{gathered}
$$

During these transmissions, the data sequences $X_{f}$ and $X_{n}$ can be detected accurately or erroneously as $\hat{X}_{f}$ and $\hat{X}_{n}$. In order to create a legitimate transmission environment for these two sets of data, the optimum detectors for both the far and near users respectively can be mathematically derived as follows:

$$
\begin{gathered}
P\left(\mathbf{X}_{\mathbf{f}}=\mathbf{X}_{\mathbf{f}} \mid \mathbf{y}_{\mathbf{f}}\right) \geq P\left(\mathbf{X}_{\mathbf{f}}=\hat{\mathbf{X}}_{\mathbf{f}} \mid \mathbf{y}_{\mathbf{f}}\right), \\
P\left(\mathbf{X}_{\mathbf{n}}=\mathbf{X}_{\mathbf{n}} \mid \mathbf{y}_{\mathbf{n}}\right) \geq P\left(\mathbf{X}_{\mathbf{n}}=\hat{\mathbf{X}}_{\mathbf{n}} \mid \mathbf{y}_{\mathbf{n}}\right) .
\end{gathered}
$$

According to these inequalities, the intervals between the $\mathbf{y}$ values and the correctly detected $\mathbf{X}$ values for both the far and near users are smaller than the $\hat{\mathbf{X}}$ values. Since there are two probabilities of detection for both the far and near receivers, their threshold can be determined by their mean values as $\frac{\mathbf{X}_{\mathbf{f}}+\hat{\mathbf{X}}_{\mathbf{f}}}{2}$ and $\frac{\mathbf{X}_{\mathbf{n}}+\hat{\mathbf{X}}_{\mathbf{n}}}{2}$ for the far and near users respectively. In this regard, the correct detection probability for the far and near users can be formulated as

$$
\begin{aligned}
& P\left(\mathbf{y}_{\mathbf{f}, \mathbf{n}}<\frac{\mathbf{X}_{\mathbf{f}, \mathbf{n}}+\hat{\mathbf{X}}_{\mathbf{f}, \mathbf{n}}}{2} \mid \mathbf{X}_{\mathbf{f}, \mathbf{n}}=\mathbf{X}_{\mathbf{f}, \mathbf{n}}\right) \\
= & P\left(\mathbf{z}>\frac{\left\|\mathbf{X}_{\mathbf{f}, \mathbf{n}}-\hat{\mathbf{X}}_{\mathbf{f}, \mathbf{n}}\right\|}{2}\right)=Q\left(\frac{\left\|\mathbf{X}_{\mathbf{f}, \mathbf{n}}-\hat{\mathbf{X}}_{\mathbf{f}, \mathbf{n}}\right\|}{2 \sqrt{N_{0} / 2}}\right),
\end{aligned}
$$

where $Q($.$) is the Q-function [41] and N_{0}=N_{0, F}$.

If equation (17) is affected by the channel coefficients that are deployed for the transmission of the far user's and the near user's data, the Q-function turns into

$$
\begin{aligned}
P\left(\mathbf{y}_{\mathbf{f}}\right. & \left.<\frac{\mathbf{X}_{\mathbf{f}}+\hat{\mathbf{X}}_{\mathbf{f}}}{2} \mid \mathbf{X}_{\mathbf{f}}=\mathbf{X}_{\mathbf{f}}\right)=Q\left(\sqrt{\frac{\left.\|\left(\mathbf{X}_{\mathbf{f}}-\hat{\mathbf{X}}_{\mathbf{f}}\right) \mathbf{h}_{\mathbf{f}}\right) \|^{2}}{2 N_{0}}}\right) \\
& =Q\left(\sqrt{\frac{\mathbf{h}_{\mathbf{f}}^{\mathbf{H}}=\left(\mathbf{X}_{\mathbf{f}}-\hat{\mathbf{X}}_{\mathbf{f}}\right)^{H}\left(\mathbf{X}_{\mathbf{f}}-\hat{\mathbf{X}}_{\mathbf{f}}\right) \mathbf{h}_{\mathbf{f}}}{2 N_{0}}}\right), \quad \text { (18) }
\end{aligned}
$$

for the far user, while it turns into the following equation for the near user:

$$
\begin{aligned}
P\left(\mathbf{y}_{\mathbf{n}}\right. & \left.<\frac{\mathbf{X}_{\mathbf{n}}+\hat{\mathbf{X}}_{\mathbf{n}}}{2} \mid \mathbf{X}_{\mathbf{n}}=\mathbf{X}_{\mathbf{n}}\right)=Q\left(\sqrt{\frac{\left\|\left(\mathbf{X}_{\mathbf{n}}-\hat{\mathbf{X}}_{\mathbf{n}}\right) \mathbf{h}_{\mathbf{n}}\right\|^{2}}{2 N_{0}}}\right) \\
& =Q\left(\sqrt{\frac{\mathbf{h}_{\mathbf{n}}^{\mathbf{H}}\left(\mathbf{X}_{\mathbf{n}}-\hat{\mathbf{X}}_{\mathbf{n}}\right)^{H}\left(\mathbf{X}_{\mathbf{n}}-\hat{\mathbf{X}}_{\mathbf{n}}\right) \mathbf{h}_{\mathbf{n}}}{2 N_{0}}}\right),
\end{aligned}
$$

where the $\mathbf{h}_{\mathbf{f}}$ and $\mathbf{h}_{\mathbf{n}}$ are the channels that are deployed for the transmission of the data of the far and near users respectively. Equation (18) and (19) can be written for the far and near users respectively as

$$
\begin{array}{r}
P\left(\mathbf{y}_{\mathbf{f}}<\frac{\mathbf{X}_{\mathbf{f}}+\hat{\mathbf{X}}_{\mathbf{f}}}{2} \mid \mathbf{X}_{\mathbf{f}}=\mathbf{X}_{\mathbf{f}}\right) \\
=Q\left(\sqrt{\frac{\mathbf{h}_{\mathbf{f}} \mathbf{H}^{\mathbf{H}} \mathbf{h}_{\mathbf{f}}}{2 N_{0}}}\right)=Q\left(\sqrt{\frac{\delta_{f}}{2 N_{0}}}\right), \\
P\left(\mathbf{y}_{\mathbf{n}}<\frac{\mathbf{X}_{\mathbf{n}}+\hat{\mathbf{X}}_{\mathbf{n}}}{2} \mid \mathbf{X}_{\mathbf{n}}=\mathbf{X}_{\mathbf{n}}\right) \\
=Q\left(\sqrt{\frac{\mathbf{h}_{\mathbf{n}}^{\mathbf{H}} B \mathbf{h}_{\mathbf{n}}}{2 N_{0}}}\right)=Q\left(\sqrt{\frac{\delta_{n}}{2 N_{0}}}\right),
\end{array}
$$

where

$$
\begin{aligned}
& \delta_{f}=\mathbf{h}_{\mathbf{f}}{ }^{\mathbf{H}} A \mathbf{h}_{\mathbf{f}}= \\
& \mathbf{h}_{\mathbf{f}}{ }^{\mathbf{H}}\left(\mathbf{X}_{\mathbf{f}}-\hat{\mathbf{X}}_{\mathbf{f}}\right)^{H}\left(\mathbf{X}_{\mathbf{f}}-\hat{\mathbf{X}}_{\mathbf{f}}\right) \mathbf{h}_{\mathbf{f}}=\left\|\left(\mathbf{X}_{\mathbf{f}}-\hat{\mathbf{X}}_{\mathbf{f}}\right) \mathbf{h}_{\mathbf{f}}\right\|^{2},
\end{aligned}
$$

$$
\begin{aligned}
& \delta_{n}=\mathbf{h}_{\mathbf{n}}{ }^{\mathbf{H}} B \mathbf{h}_{\mathbf{n}}= \\
& \mathbf{h}_{\mathbf{n}}{ }^{\mathbf{H}}\left(\mathbf{X}_{\mathbf{n}}-\hat{\mathbf{X}}_{\mathbf{n}}\right)^{H}\left(\mathbf{X}_{\mathbf{n}}-\hat{\mathbf{X}}_{\mathbf{n}}\right) \mathbf{h}_{\mathbf{n}}=\left\|\left(\mathbf{X}_{\mathbf{n}}-\hat{\mathbf{X}}_{\mathbf{n}}\right) \mathbf{h}_{\mathbf{n}}\right\|^{2},
\end{aligned}
$$

and the $A$ matrix equals to $\left(\mathbf{X}_{\mathbf{f}}-\hat{\mathbf{X}}_{\mathbf{f}}\right)^{H}\left(\mathbf{X}_{\mathbf{f}}-\hat{\mathbf{X}}_{\mathbf{f}}\right)$, the $B$ matrix equals to $\left(\mathbf{X}_{\mathbf{n}}-\hat{\mathbf{X}}_{\mathbf{n}}\right)^{H}\left(\mathbf{X}_{\mathbf{n}}-\hat{\mathbf{X}}_{\mathbf{n}}\right)$.

\section{POWER CONSUMPTION}

In OFDM-SNM, the power consumption performance of the system achieves better efficiency than conventional OFDM since only the active subcarriers for the modulated data are utilized. However, in the proposed multi-user OFDM-SNM scheme, all the subcarriers are activated, since some of them, which are defined by the SNM mapper, are deployed for the far user with high power, the remaining subcarriers in the system are deployed for the transmission of the near user's 
modulated data with low power. In conventional OFDM, the transmission power $\left(P_{t x}\right)$ is distributed for all subcarriers in equal amounts, which leads the average power to be $\frac{P_{t x}}{N_{F}}$. In the proposed multi-user scheme, the possibilities of the subcarrier activation for each subblock are given for the far and near users respectively as

$$
\begin{array}{r}
P_{h}\left(N_{a c}=K\right)=\left(\begin{array}{c}
N \\
K
\end{array}\right) p_{a}^{K}\left(1-p_{a}\right)^{N-K}, \\
P_{l}\left(N_{a c}=K\right)=\left(\begin{array}{c}
N \\
N-K
\end{array}\right) p_{b}^{N-K}\left(1-p_{b}\right)^{K},
\end{array}
$$

where $N_{a c}$ is the number of active subcarriers, which varies as $K$ s possible values vary from 1 to $N, p_{a}$ is the probability that $N_{a c}=K$, while $p_{b}$ is the probability that $N_{a c}=N-K$.

In this regard, the power consumption of the far user can be formulated as

$$
P_{f}=\frac{P_{t x}}{G N} \sum_{g=1}^{G} K(g) P\left(N_{a c}=K(g)\right),
$$

while the power consumption of the near user is formulated as

$$
P_{n}=\frac{P_{t x}}{G N} \sum_{g=1}^{G}(N-K)(g) P\left(N_{a c}=(N-K)(g)\right),
$$

where $P_{t x}$ represents the total power of transmission.

After each user's consumed power is found, the total power consumption of the system, $P_{T}$, can be found as the summation of the equations (26) and (27) stated as follows:

$$
P_{T}=P_{f}+P_{n} .
$$

\section{SIMULATION RESULTS}

In this section, the simulation results of the proposed multiuser OFDM-SNM are presented in terms of the bit error rate (BER) and throughput. The simulation parameters are

\begin{tabular}{|c|c|}
\hline Modulation Type & $\operatorname{BPSK}(M=2)$ \\
\hline IFFT/FFT Size $\left(N_{F}\right)$ & 64 \\
\hline CP Guard Interval (Samples) & 8 \\
\hline Number of Subblocks in Each OFDM Symbol (G) & 16 \\
\hline Number of Available Subcarriers in Each Subblock (N) & 4 \\
\hline Number of Bits Mapped to Each Subblock $\left(p_{1}\right)$ & 2 \\
\hline Multipath Channel Delay Samples Locations & 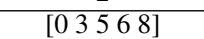 \\
\hline Multipath Channel Tap Power Profile (dBm) & {$\left[\begin{array}{lllll}0 & -8 & -17 & -2 & -25\end{array}\right]$} \\
\hline
\end{tabular}
provided in Table II. For the simulated multi-user OFDMSNM, the multipath channel is assumed to be Rayleigh distributed.

TABLE 2. Simulation Parameters

Fig. 3 depicts the BER performances of the far and near users. It can be seen from the figure that while the far user gets better results in terms of BER, the near user does not achieve the same performance, since in some cases, where all the subcarriers are activated for the far user, the near user is not served.

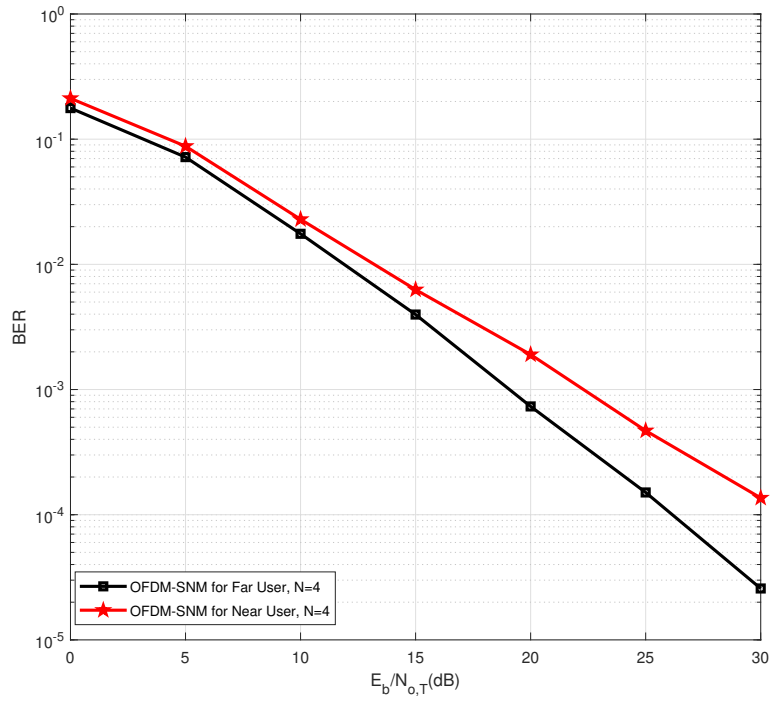

FIGURE 3. BER comparison of proposed multi-user OFDM-SNM between the Far User and Near User

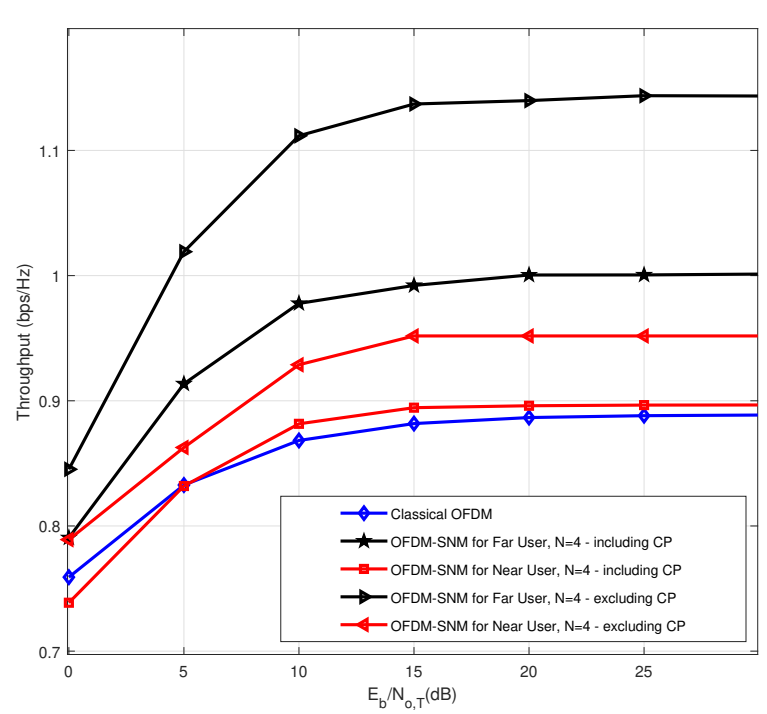

FIGURE 4. Throughput comparison of proposed multi-user OFDM-SNM between the Far User, Near User, and Conventional OFDM Under BPSK.

Fig. 4 shows the throughput performances of the far and near users in both cases where the $\mathrm{CP}$ is included and excluded. Furthermore, the simulation results are compared with the throughput result of conventional OFDM. It can be inferred from the figure that the throughput performance achieved by the near user shows a similar performance with conventional OFDM, as in each of the time only one symbol is transmitted to the near user while the throughput performance of the far user outperforms the near user's 
performance since it carries the characteristics of SNM.

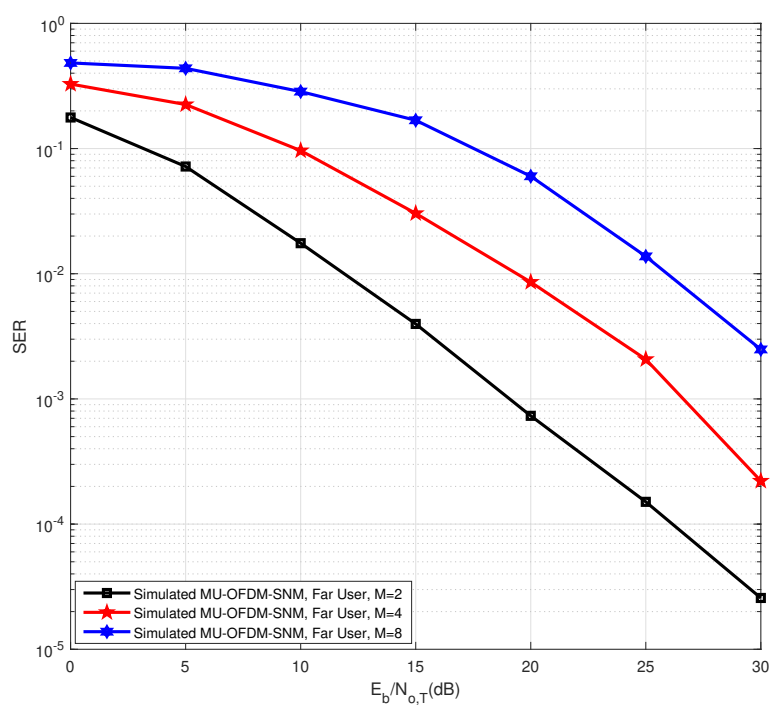

FIGURE 5. BER comparison of proposed multi-user OFDM-SNM for the Far User under different $M$-ary modulation orders.

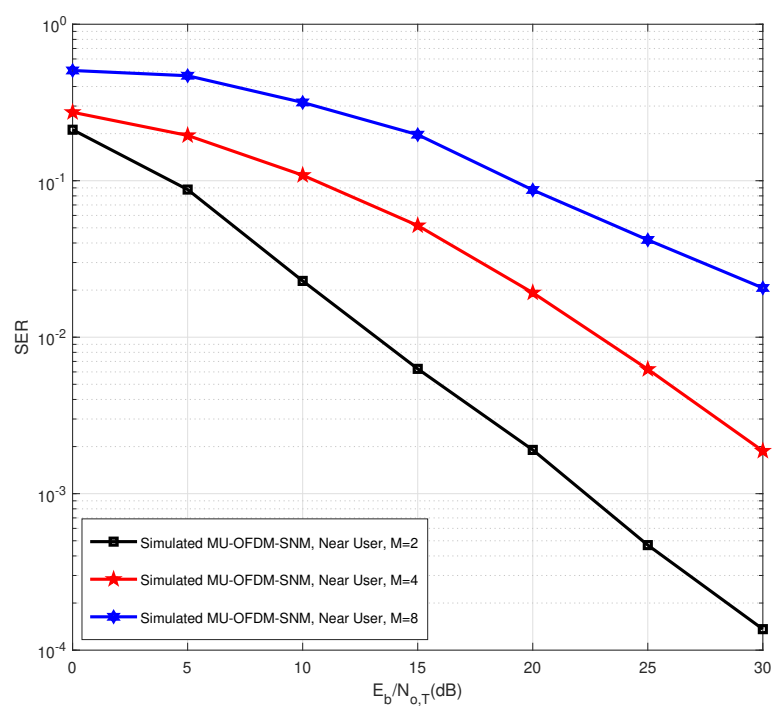

FIGURE 6. BER comparison of proposed multi-user OFDM-SNM for the Near User under different $M$-ary modulation orders.

Fig. 5 and Fig. 6 show the simulation performances of the proposed multi user OFDM-SNM scheme under different $M$-ary modulation orders for the far user and near user respectively. It can be inferred from these figures that while the SER performance of the far user exhibits the best performance under BPSK $(M=2)$, its performance degrades as the modulation order gets higher. However, for the case where the QPSK ( $M=4$ ) modulation is used for the

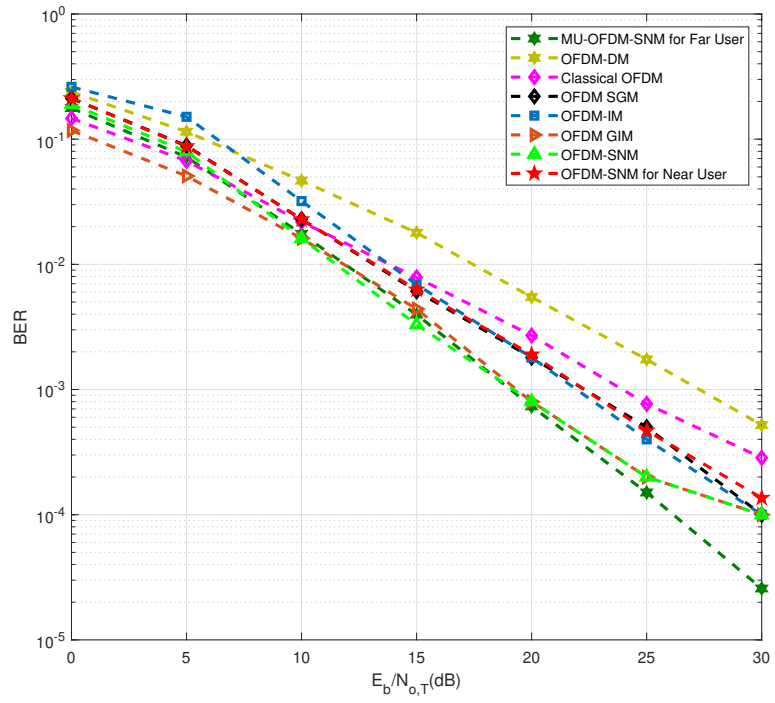

FIGURE 7. BER comparison of the far user, near user and other schemes in the literature.

transmission of the far user, it can be inferred from the Fig. 5 and Fig. 6 that the SER performance of the far user achieves almost the same performance of the BER performance of the near user where the selected modulation order is BPSK for the transmission of the near user's data.

It also should be noted that, since the first priority of the proposed multi-user adapted OFDM-SNM scheme is the transmission of the far user's data, where in some cases the near user is not served, there is not any compromise in the BER performance of the far user. In fact, it shows the same performance as the single user OFDM-SNM. Fig. 7 shows the BER comparison of the multi-user OFDM-SNM with other schemes in the literature. The output result of the proposed scheme for the far user exhibits a similar performance as OFDM-IM, and surpasses conventional OFDM as well as other techniques such as OFDM-SGM and OFDM-DM. As it can be interpreted from this figure, the proposed technique has the capability of serving another user without degrading the BER performance of the main user.

\section{CONCLUSION}

In this paper, a novel multi-user transmission technique is introduced by inheriting the features of OFDM-SNM. By applying this technique, it is aimed to serve another user who is located in a near location by utilizing the initially unemployed subcarriers that remain from the data transmission of the primary user who is located in a far location, while OFDM-SNM is applied to transmit data bits by exploiting the number of active subcarriers in each subblock. Simulations and mathematical performance analysis prove that the proposed multi-user OFDM-SNM provides better BER performance for the users, who are situated in a nearer location 
from the transmitter than conventional OFDM, since the data transmission is operated by multiple subcarriers. It is also proven that the transmission of data that adapts OFDM-SNM for a user who is situated on a far location from the transmitter does not need to compromise in terms of performance to achieve this secondary gain, since the characteristics of the proposed scheme are the same as the single user OFDMSNM. With these merits of the proposed multi-user OFDMSNM scheme, it can be said that by deploying the inactive subcarriers that are not deployed for the transmission of the data of the far user in each subblock for the near user, more devices can be served in environments where the data traffic is high, and additionally, the spectrum can be utilized more efficiently by exploiting the simultaneous data service. All this makes the proposed scheme a strong candidate to be used in future $6 \mathrm{G}$, and beyond technologies.

\section{REFERENCES}

[1] J. M. Hamamreh, M. Kirik, M. O. Sagman, and N. Ishikawa, "Multiple input multiple output with antenna number modulation and adaptive antenna selection," RS Open Journal on Innovative Communication Technologies, vol. 1 , no. $1,2020$.

[2] M. Kirik and J. M. Hamamreh, "Multiple mimo with joint block antenna number modulation and adaptive antenna selection for future wireless systems," RS Open Journal on Innovative Communication Technologies, vol. 1 , no. 2 , p. $12,2020$.

[3] A. A. Zaidi, R. Baldemair, H. Tullberg, H. Bjorkegren, L. Sundstrom, J. Medbo, C. Kilinc, and I. Da Silva, "Waveform and numerology to support 5g services and requirements," IEEE Communications Magazine, vol. 54 , no. 11 , pp. $90-98,2016$

[4] A. M. Jaradat, J. M. Hamamreh, and H. Arslan, "Ofdm with hybrid number and index modulation," IEEE Access, vol. 8, pp. 55 042-55 053, 2020.

[5] J. Armstrong, "Ofdm for optical communications," Journal of lightwave technology, vol. 27, no. 3, pp. 189-204, 2009.

[6] J. Jang and K. B. Lee, "Transmit power adaptation for multiuser ofdm systems," IEEE Journal on selected areas in communications, vol. 21, no. 2, pp. 171-178, 2003.

[7] X. Wang, P. Ho, and Y. Wu, "Robust channel estimation and isi cancellation for ofdm systems with suppressed features," IEEE Journal on Selected Areas in Communications, vol. 23, no. 5, pp. 963-972, 2005.

[8] H. Sampath, S. Talwar, J. Tellado, V. Erceg, and A. Paulraj, "A fourthgeneration mimo-ofdm broadband wireless system: design, performance, and field trial results," IEEE Communications Magazine, vol. 40, no. 9, pp. 143-149, 2002.

[9] J.-J. Van De Beek, O. Edfors, M. Sandell, S. K. Wilson, and P. O Borjesson, "On channel estimation in ofdm systems," in 1995 IEEE 45th Vehicular Technology Conference. Countdown to the Wireless TwentyFirst Century, vol. 2. IEEE, 1995, pp. 815-819.

[10] R. D. Van Nee and R. Prasad, "Ofdm for wireless multimedia communications," 2000.

[11] Z. Shen, J. G. Andrews, and B. L. Evans, "Adaptive resource allocation in multiuser ofdm systems with proportional rate constraints," IEEE transactions on wireless communications, vol. 4, no. 6, pp. 2726-2737, 2005.

[12] A. Sahin, I. Guvenc, and H. Arslan, "A survey on multicarrier communications: Prototype filters, lattice structures, and implementation aspects," IEEE communications surveys \& tutorials, vol. 16, no. 3, pp. 1312-1338, 2013.

[13] T. Hwang, C. Yang, G. Wu, S. Li, and G. Y. Li, "Ofdm and its wireless applications: A survey," IEEE transactions on Vehicular Technology, vol. 58, no. 4, pp. 1673-1694, 2008.

[14] M. Vaezi, Z. Ding, and H. V. Poor, Multiple access techniques for 5G wireless networks and beyond. Springer, 2019, vol. 159.

[15] A. M. Jaradat, J. M. Hamamreh, and H. Arslan, "Floating ofdm-snm for papr and oobe reduction," in 2021 IEEE 93rd Vehicular Technology Conference (VTC2021-Spring). IEEE, 2021, pp. 1-5.

[16] — "Modulation options for ofdm-based waveforms: Classification, comparison, and future directions," IEEE Access, vol. 7, pp. $17263-$ $17278,2019$.
[17] E. Başar, Ü. Aygölü, E. Panayırc1, and H. V. Poor, "Orthogonal frequency division multiplexing with index modulation," IEEE Transactions on signal processing, vol. 61, no. 22, pp. 5536-5549, 2013.

[18] Y. Belallou, J. M. Hamamreh, and A. Hajar, "Ofdm-subcarrier power modulation with two dimensional signal constellations," in 2019 Innovations in Intelligent Systems and Applications Conference (ASYU). IEEE, 2019, pp. 1-6.

[19] J. M. Hamamreh, A. Hajar, and M. Abewa, "Orthogonal frequency division multiplexing with subcarrier power modulation for doubling the spectral efficiency of $6 \mathrm{~g}$ and beyond networks," Transactions on Emerging Telecommunications Technologies, vol. 31, no. 4, p. e3921, 2020.

[20] A. M. Jaradat, J. M. Hamamreh, and H. Arslan, "Ofdm with subcarrier number modulation," IEEE Wireless Communications Letters, vol. 7 no. 6, pp. 914-917, 2018.

[21] E. Başar, "Multiple-input multiple-output ofdm with index modulation,' IEEE Signal Processing Letters, vol. 22, no. 12, pp. 2259-2263, 2015.

[22] J. M. HAMAMREH and A. HAJAR, "The generalization of orthogonal frequency division multiplexing with subcarrier power modulation to quadrature signal constellations," RS Open J. Innov. Commun. Technol, vol. 4, pp. 1-15, 2020.

[23] M. Abewa and J. M. Hamamreh, "Non-coherent ofdm-subcarrier power modulation for low complexity and high throughput iot applications," RS Open Journal on Innovative Communication Technologies, vol. 1, no. 1, 2020.

[24] — , "Nc-ofdm-spm: a two-dimensional non-coherent modulation scheme for achieving the coherent performance of ofdm along with sending an additional data-stream," 2021.

[25] M. Hijazi and J. M. Hamamreh, "Convolutional neural network based equalizer for improving the reliability performance of ofdm with subcarrier power modulation,” RS Open Journal on Innovative Communication Technologies, vol. 2, no. 5, 7 2021, https://rs-ojict.pubpub.org/pub/fch9opwb. [Online]. Available: https://rs-ojict.pubpub.org/pub/fch9opwb

[26] A. Abuqamar and J. M. Hamamreh, "Back propagation artificial neural network for improving the performance of stbc-based ofdm with subcarrier power modulation," RS Open Journal on Innovative Communication Technologies, vol. 2, no. 4 , 6 2021, https://rs-ojict.pubpub.org/pub/wc360yx6. [Online]. Available: https://rs-ojict.pubpub.org/pub/wc360yx6

[27] A. Abuqamar, J. M. Hamamreh, and M. Abewa, "Stbcassisted ofdm with subcarrier power modulation," RS Open Journal on Innovative Communication Technologies, vol. 2, no. 4 5 2021, https://rs-ojict.pubpub.org/pub/50bbe9c1. [Online]. Available: https://rs-ojict.pubpub.org/pub/50bbe9c1

[28] S. Dang, G. Ma, B. Shihada, and M.-S. Alouini, "Enhanced orthogonal frequency-division multiplexing with subcarrier number modulation," IEEE Internet of Things Journal, vol. 6, no. 5, pp. 7907-7920, 2019.

[29] J. Li, S. Dang, M. Wen, Z. Zhang, and Q. Li, "Smart detection using the cascaded artificial neural network for ofdm with subcarrier number modulation," IEEE Wireless Communications Letters, vol. 10, no. 6, pp. $1227-1231,2021$

[30] S. Dang, J. Zhou, B. Shihada, and M.-S. Alouini, "Relay assisted ofdm with subcarrier number modulation in multi-hop cooperative networks," IEEE Wireless Communications Letters, vol. 9, no. 11, pp. 1869-1873, 2020.

[31] S. Dang, S. Guo, B. Shihada, and M.-S. Alouini, "Information-theoretic analysis of ofdm with subcarrier number modulation," IEEE Transactions on Information Theory, 2021.

[32] J. Li, S. Dang, M. Wen, S. Mumtaz, Q. Li, and C. X. Mavromoustakis, "Hybrid orthogonal frequency division multiplexing with subcarrier number modulation," in ICC 2021-IEEE International Conference on Communications. IEEE, 2021, pp. 1-6.

[33] E. Başar, "Ofdm with index modulation using coordinate interleaving," IEEE Wireless Communications Letters, vol. 4, no. 4, pp. 381-384, 2015.

[34] R. Abu-Alhiga and H. Haas, "Subcarrier-index modulation ofdm," in 2009 IEEE 20th International Symposium on Personal, Indoor and Mobile Radio Communications. IEEE, 2009, pp. 177-181.

[35] D. Tsonev, S. Sinanovic, and H. Haas, "Enhanced subcarrier index modulation (sim) ofdm," in 2011 IEEE GLOBECOM Workshops (GC Wkshps). IEEE, 2011, pp. 728-732.

[36] M. Wen, J. Li, S. Dang, Q. Li, S. Mumtaz, and H. Arslan, "Joint-mapping orthogonal frequency division multiplexing with subcarrier number modulation," IEEE Transactions on Communications, 2021. 
[37] S. Karatepe, M. Kirik, and J. M. Hamamreh, "Novel nonorthogonal multiaccess method for multi-user mimo with antenna number modulation," RS Open Journal on Innovative Communication Technologies, vol. 2, no. 3, 2021.

[38] A. Jaradat, J. M. Hamamreh, and H. Arslan, "Orthogonal frequency division multiplexing with subcarrier gap modulation," in 2020 IEEE 31st Annual International Symposium on Personal, Indoor and Mobile Radio Communications. IEEE, 2020, pp. 1-6.

[39] M. E. Sahin, I. Guvenc, and H. Arslan, "Optimization of energy detector receivers for uwb systems," in 2005 IEEE 61st Vehicular Technology Conference, vol. 2. IEEE, 2005, pp. 1386-1390.

[40] M. Wen, E. Basar, Q. Li, B. Zheng, and M. Zhang, "Multiple-mode orthogonal frequency division multiplexing with index modulation," IEEE Transactions on Communications, vol. 65, no. 9, pp. 3892-3906, 2017.

[41] M. Chiani and D. Dardari, "Improved exponential bounds and approximation for the q-function with application to average error probability computation," in Global Telecommunications Conference, 2002. GLOBECOM'02. IEEE, vol. 2. IEEE, 2002, pp. 1399-1402.

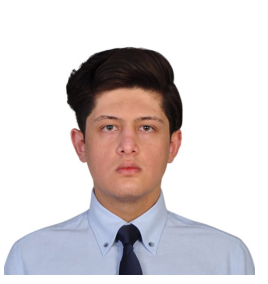

MUHAMMET KIRIK received the B.Sc. degree in electrical and electronics engineering from Antalya Bilim University, Turkey in 2020. His current research interests include orthogonal frequency division multiplexing multiple input multiple output systems, multi-dimensional modulation techniques, and orthogonal/non-orthogonal multiple access schemes for future wireless systems.

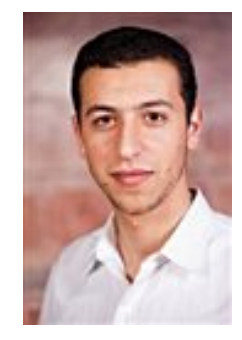

JEHAD M. HAMAMREH received the B.Sc. degree in electrical and telecommunication engineering from An-Najah University, Nablus, in 2013, and the Ph.D. degree in electrical electronics engineering and cyber systems from Istanbul Medipol University, Turkey, in 2018. He was a Researcher with the Department of Electrical and Computer Engineering, Texas AM University. He is currently an Assistant Professor with the Electrical and Electronics Engineering Department, Antalya International (Bilim) University, Turkey. His current research interests include wireless physical and MAC layers security, orthogonal frequency-division multiplexing multiple-input multiple-output systems, advanced waveforms design, multi-dimensional modulation techniques, and orthogonal/non-orthogonal multiple access schemes for future wireless systems. He is a Regular Reviewer for various peer-reviewed journals as well as a TPC Member for several international conferences. He is very open for collaboration and can be reached via email at jehad.hamamreh@gmail.com. 